manifestations of infection such as fever, rigors, and/or hypotension and a positive blood cultures obtained via CVC in the absence of other potential sources of infection. CVC were removed if severe, potentially life-threatening symptoms occurred. The incidence of CRBSI was measured as number of catheter-related episodes per 1000/catheter days.

Results A total of 58 children (26 male, aged $7.2 \pm 4.6$ years) were reviewed. The indications for PN were motility disorder in $44.8 \%$, short bowel syndrome in $36.2 \%$ and enteropathy in $19 \%$. The catheters used were single-lumen tunneled Hickmann (82/108), double-lumen (26/108), peripheral inserted central catheter $(2 / 108)$ and Broviac (1/108).

Thirty-one of $58(53.4 \% ; 15 \mathrm{M}$, aged $5.8 \pm 4.3$ years $)$ children developed 108 CRBSIs over the study period. The median (range) number of CRBSI episodes per patient was 1 (0-14). The overall catheter days was 58414 and the CRBSI rate was $1.85 / 1000$ catheter days.

Only $23(21.3 \%)$ catheters were removed because of lifethreatening symptoms and $85(78.7 \%)$ of catheters were salvaged and retained despite CRBSI.

By organism, 38\% were gram positive, 34.2\% gram negative, $21.2 \%$ polymicrobial and $6.5 \%$ fungal CRBSI. The most frequent gram positive and negative organism was Staphylococcus aureus (31.7\%) and Klebsiella species (43.2\%) respectively. Catheter infected with gram positive bacteria showed the highest rate of CVC salvage (gram positive 92.7\%, 78.2\% polymicrobial, $67.6 \%$ gram negative, $57.1 \%$ fungal infection; $\mathrm{P}<0.05)$.

The CRBSI rate for double-lumen catheters was significantly greater than single-lumen catheters $(24.1 \%$ vs $4.8 \%$; $\mathrm{P}<0.0001)$. Patients with a double-lumen CVC were found to be at increased risk for CRBSI development (HR 2.51; [95\% CI 1.70-3.86]; P <0.01).

Conclusion CVC is possible in more than three-quarters of CRBSIs in children on long-term home PN for IF. Successful salvage may depend on the species isolated. CRBSIs caused by gram positive bacteria, the most bacteria causing CRBSI, had a CVC salvage rate approaching 93\%. Effective antibiotic treatment without removal of the CVC should be considered as first line treatment. A single-lumen CVC should be the catheter of first choice. Further studies to identify predictive factors of catheter removal after CRBSI are required.

\section{P53 THE EFFECTIVENESS OF COLONIC TRANSIT STUDIES IN THE OPTIMISATION OF THE MANAGEMENT OF CHRONIC CONSTIPATION}

Matthew Gould, Elizabeth Renji, Raj Parmar. Alder Hey Children's Hospital

10.1136/flgastro-2021-bspghan.62

Introduction/Background Chronic constipation has been shown to lead to poor school performance and consequently deficiencies in education, as well as poor health-related quality of life. In children who suffer from chronic constipation, colonic transit studies (CTS) are ordered by specialist services to provide information that aids clinical management decisions

Aim The aim of this audit was to evaluate the impact of CTS outcomes on clinical management decisions involving patients with chronic constipation. It also looked at the radiology reports of included transit studies, specifically at whether they included the number and location of radio opaque markers. The NICE guideline 'Constipation in children and young people: diagnosis and management' and The Royal College of Radiologists audit template 'Complete reporting of colonic transit marker studies' were used to determine best practice.

Subjects and Method A retrospective audit looking at the list of patients with chronic constipation who underwent CTS at Alder Hey Children's Hospital. Working backwards from November 2019, the first 100 patients who met inclusion criteria were selected. Included patients had to best knowledge conducted CTS in full and also had a clinic letter following completion of the study. Management outcomes were grouped into 4 categories: decrease, no change to management, an increase of oral laxatives or an increase using management stronger than oral laxatives e.g. rectal medications or surgical interventions.

Results The majority of included transit studies were requested by either paediatric surgery $(n=71)$ or gastroenterology $(n=20)$. Only $60 \%$ of CTS reports included both the number and location of markers and 13\% included neither. There was a mean of 8 days from transit study to radiology report completion. The mean transit time was 72 hours, with a range of 0-144 hours. Management outcomes were varied for both normal and slow transit. Twice as many patients with slow transit were managed with therapies stronger than oral laxatives. Patients with normal transit time were over twice as likely to have no change to their management. A transit time of $>100$ hours resulted in almost $80 \%$ of patients being managed with treatment stronger than oral laxatives.

Summary and Conclusion There appears to be a trend towards escalating management with intensive combination treatment regimes in patients whose CTS suggested slow transit and especially in patients with transit times greater than 100 hours. The range of the management choices used in patients with normal transit do however illustrate that clinicians within Alder Hey are making clinical decisions based upon the wider clinical picture of the patient, which fits with NICE guidance. This audit does illustrate that CTS radiology reports can be adapted to ensure each report contains the number and location of markers.

Recommendations All CTS radiology reports should include the number and location of radio opaque markers. The location of markers should be reported into 3 regions (right colon, left colon and rectosigmoid colon) as suggested. A proforma has been distributed within the Alder Hey radiology department detailing results and recommendations. A re-audit to assess the application of these recommendations is currently underway.

\section{P54 THE GASTROINTESTINAL PRESENTATION OF PIMS-TS/ MIS-C IN A COHORT AT A TERTIARY CENTRE PAEDIATRIC CENTRE}

Elena Kurteva*, Tania Ahmad*, Karlie Grant, Justin Penner, Fevronia Kiparissi, Edward Peter Gaynor. Great Ormond Street Hospital; * Joint first author

\subsection{6/flgastro-2021-bspghan.63}

Introduction/Background Paediatric inflammatory multisystem syndrome temporally associated with COVID-19 (PIMS-TS) is a novel condition with poorly understood pathophysiology. Acute presentation varies, with some children acutely 